\title{
Normes et transgression au XVIII siècle. Textes réunis par Pierre Dubois
}

\section{Gianmaria Zamagni}

\section{Q OpenEdition}

1 Journals

\section{Edizione digitale}

URL: https://journals.openedition.org/studifrancesi/39353

DOI: 10.4000/studifrancesi.39353

ISSN: 2421-5856

\section{Editore}

Rosenberg \& Sellier

\section{Edizione cartacea}

Data di pubblicazione: 1 décembre 2004

Paginazione: 366-367

ISSN: 0039-2944

\section{Notizia bibliografica digitale}

Gianmaria Zamagni, «Normes et transgression au XVIII" siècle. Textes réunis par Pierre Dubois», Studi Francesi [Online], 143 (XLVIII | II) | 2004, online dal 30 novembre 2015, consultato il 19 mai 2021. URL: http://journals.openedition.org/studifrancesi/39353; DOI: https://doi.org/10.4000/studifrancesi. 39353

Questo documento è stato generato automaticamente il 19 mai 2021.

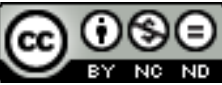

Studi Francesi è distribuita con Licenza Creative Commons Attribuzione - Non commerciale - Non opere derivate 4.0 Internazionale. 


\title{
Normes et transgression au XVIII siècle. Textes réunis par Pierre Dubois
}

\author{
Gianmaria Zamagni
}

\section{NOTIZIA}

Normes et transgression au XVIII ${ }^{e}$ siècle. Textes réunis par Pierre DUBoIs, Groupe interdisciplinaire d'étude du XVIII ${ }^{\mathrm{e}}$ siècle de l'Université Blaise-Pascal (Clermont II), Paris, Presses de l'Université de Paris-Sorbonne, 2002, pp. 250.

1 Il testo è frutto dello studio della nozione di trasgressione a cui si è dedicato il Groupe dell'Università Blaise-Pascal di Clermont-Ferrand. Si tratta di un volume ricco di quindici agili contributi che spaziano dalle scienze politiche e sociali all'aspetto storicoreligioso, fino al côté estetico della nozione presa in oggetto. Il XVIII è «secolo di rivoluzioni», eppure, come precisa Pierre DUBoIs nell'Introduction, al tempo delle Lumières, è innanzi tutto una teoria della legge naturale a permettere la critica dei poteri stabiliti, poiché sono questi ultimi a trasgredire l'ideale di tolleranza che promana da quella legge. Non si tratta allora di una sovversione, ma di un «rejet des formalismes et des lois arbitraires» (p. 14), per fare in modo che non sia tanto l'uomo a doversi adattare alla regola, quanto questa a quello. Il contributo di Philippe BOURDIN, Révolution et transgression: autour de la mort du roi (pp. 15-36), apre il volume per la domanda che pone, di carattere metodologico: può il concetto di trasgressione rappresentare uno strumento efficace di analisi storica? Questa domanda trova risposta nell'articolazione del tema del regicidio (con utili riferimenti al mutamento dell'iconografia nel corso della Rivoluzione):

2 Lungi dall'essere «moteur de l'histoire», la trasgressione può rappresentare una «façon subjectivement raisonnée de la vivre et de l'interpréter» (p. 33). Nello stesso orizzonte della Introduction di Dubois, Jean EHRARD legge il capolavoro montesquieuiano nel suo 
Normes et transgression dans «L'esprit des Lois» (pp. 37-44). Fin dal primo libro dello Spirito delle leggi, ciò che soggiace alle leggi umane è l'immutabile legge naturale, ed è ancora per Montesquieu la saggezza del Creatore a fondare e garantire l'ordine della creazione. Al di sotto di questo piano, tuttavia, il Bordolese riconosce quell'universo fatto dall'umanità fallibile, retto da altre leggi. Esse devono obbedire in primo luogo alla ragione, ed è in questo senso che - al di là della trasgressione delle norme del diritto penale (e l'attenzione è qui volta alle più contestate: su magia, eresia, omosessualità $\mathrm{e}$ lesa maestà) - si può vedere in filigrana un'altra, peggiore trasgressione, quella costituita da leggi dispotiche, false norme, e dato che «la loi n'est pas un pur acte de puissance[...] les choses indifférentes par leur nature ne sont pas de son ressort» (Esprit des Lois, XII, 11). Nell'articolo di Daniel MARTIN, infine, Transgression sociale et arbitraire monarchique: le recours aux lettres du roi (pp. 45-52), si mostrano i molti casi nei quali la risposta dell'amministrazione reale alle lettere di privati e curati, volte ad arginare la trasgressione delle loro regole, si dimostrava assai attenta alla libertà individuale, e questo poiché anche gli intendenti della corona erano di fatto uomini delle Lumières.

Segue questi contributi l'ampia sezione dedicata alla tematica in oggetto in violazione delle norme religiose: dapprima Danielle CORRADO studia il caso di José-Maria Blanco White: un exemple de transgression politique et religieuse en Espagne (pp. 53-67), sottolineando la responsabilità che il curato attribuisce alla religione cattolica nella distruzione delle libertà individuali. Questo atteggiamento costringerà Blanco White alla fuga dalla Spagna, dalla propria religione e financo dalla propria identità, in nome di un'instancabile lotta contro la tirannia della religione e del potere. Successivamente è il caso dell'abbé ribelle Marchena a essere fatto oggetto di studio nel contributo di Evelyne MARTIN-HERNANDEZ, l'abbé Marchena ou la transgression faite homme, selon Menéndez y Pelayo (pp. 69-77). Jacques CARRÉ invece dedica la propria attenzione agli ospedali e alle loro modalità repressive in Le désordre et la règle: la gestion des comportements "déviants» dans quelques établissements charitables anglais au XVIII siècle (pp. 79-96), rilevando come la trasgressione dell'ordine ospedaliero rappresentasse una vera e propria «revendication identitaire» dei poveri (p. 94) di fronte alle richieste talora incomprensibili dei «benefattori». In seguito Stéphane GomIs, con il contributo La communauté de prêtres dans la France du XVIII siècle: un clergé en-dehors de la norme? (pp. 97-116), studia il caso dei preti «comunalisti» nella Francia dell'Ancien Régime, osservando quanto aumentata fosse la capacità di adattamento del clero alla realtà sociale rispetto al secolo precedete. Anthony McKenNA, infine, conclude questa ampia serie con il suo La norme et la transgression: Pierre Bayle et le socinianisme (pp. 117-136). ̇̀ Bayle stesso a fornire una definizione dei sociniani come «coloro che non credono che sia rivelazione tutto ciò che non è conforme alle massime della ragione e all'esperienza della natura»; è chiara in questo senso la riduzione della rivelazione alla filosofia. Eppure, sebbene lo stesso Bayle in apertura del Commentaire philosophique dichiarasse come propria questa medesima posizione, nel recensire un testo sociniano come la Religio naturalis di Andreas Wiszowatius, egli ritiene che l'autore non segua coerentemente le esigenze di questi principi, operando una distinzione (che Bayle rigetta) fra ciò che sta al di là e ciò che sta contro la ragione. Se questa separazione non è legittima, come argomenta Bayle, non è possibile alcuna conciliazione fra fede e ragione; e così, lo stesso che fondava il proprio Commentaire philosophique sul razionalismo morale sociniano giunge a dichiarare come occorra abbandonare ogni ragione per credere alla dottrina cristiana. Quello che obietta al sociniano non è tanto il suo appello alla ragione, quanto il compromesso che egli cerca per conciliare fra fede e 
ragione. La trasgressione di cui si tratta qui, dunque, è quella sociniana, respinta perché non permette al razionalismo di giungere alla sua logica, scettica conclusione.

La restante parte del volume prende in oggetto la trasgressione nella sua accezione estetica: Friederike SPITZL-DUPIC si occupa delle Transgressions privées et publiques chez Georg Christoph Lichtenberg (pp. 137-162), illuminista che ne fa proprio un «principe intime de réflexion et d'écriture» (p. 161) in triplice maniera; trasgressioni in primo luogo verso l'estemo: politiche, sociali, religiose e morali; in secondo luogo interne, intellettuali, volte ad aumentare le conoscenze, la comprensione del mondo e dell'uomo; autocontraddittorie in qualche modo, infine, quando sconfinano nell'irrazionale, nel mostruoso, persino nel deleterio Lucette BEROL, ne «La religieuse» et la dynamique de la transgression (pp. 163-177) legge il romanzo di Diderot per vedervi come per l'encyclopédiste ogni trasgressione susciti sempre nuovi trasgressioni, finendo per contagiare quella stessa forma letteraria. Pierre Dubois e Gérard Loubinoux, successivamente, prendono in considerazione l'estetica musicale, rispettivamente con $\mathrm{i}$ loro contributi Musique et idéologie en Angleterre au XVIII siècle: la peur de la transgression (pp. 179-196) e Mesure et expression en musique, ou Rousseau et la transgression interdite (pp. 197-208), sottolineando come nonostante il rifiuto di trasgressioni alla misura e alla nozione di espressione, come nell'arte dei giardini, venga rigettato il formalismo rigido, quasi un'ennesima «fausse norme» nell'accezione montesquieuiana. Jean DELINIÈRE, con il proprio «Les souffrances du jeune Werther», un batard de génie sur la scène romanesque de 1774 (pp. 209-217), permette di comprendere come proprio la trasgressione sia centrale nel passaggio a una nuova età: il Werther, e segnatamente il suo suicidio (gesto trasgressivo per eccellenza), mostrano il contrapporsi delle regole sociali alla natura, e assieme già annunciano il Romanticismo. All'inizio del nuovo secolo, la trasgressione prende un'altra forma. Come sostiene Marion MARCEAU nel proprio contributo Kruitzner: expansion des frontières de la transgression (pp. 219-231), se, da una parte, Harriet Lee (autrice del Kruitzner) non mette in pratica le idee sulla trasgressione che si sviluppano nel romanzo, sarà viceversa Byron a spingerle all'estremo, divenendo l'incarnazione stessa della trasgressione, col suo adattamento del romanzo nel Werner. Lucette PEROL, infine, con L'exploitation philosophique de la transgression: l'épisode de Butua dans "Aline et Valcour» de Sade (pp. 234-247), osserva quanto il romanziere spinga l'ideologia delle Lumières fino alle loro estreme conseguenze, attraverso un romanzo che intende mettere in campo ogni trasgressione. 